\title{
A comparison of results of SRS-30 questionnaire in scoliosis patients treated surgically or conservatively
}

H Yilmaz ${ }^{1 *}$, T Kuru ${ }^{2}$

From 8th International Conference on Conservative Management of Spinal Deformities and SOSORT 2011

Annual Meeting

Barcelona, Spain. 19-21 May 2011

\section{Background}

The Scoliosis Research Society Questionnaire (SRS-30) is a specific instrument to measure health-related quality of life in patients with scoliosis who had undergone surgery or had not had. The objectives of this paper are evaluate pain, function, self-image, mental health and satisfaction with management of scoliosis patients and compare of surgical and conservative treatment.

\section{Materials and methods}

The questionnaire was fulfilled by 80 individuals with scoliosis on the internet and we evaluated their answers. The mean age of the participants at the time they received the questionnaire was $18.6 \pm 4,2$ years (min:12years, max:36years). 35 patients were treated with surgery and 45 were treated with conservative management (brace or/and exercise). 18 individuals were male and 62 individuals were female. Data analysis were performed using SPSS, version 12.

\section{Results}

There were no differences between pain, function, selfimage, mental health and satisfaction with management scores of patients who had surgery or not had (Table 1).

\section{Conclusions}

The data obtained in this study there were no significant differences in pain, function, self-image, mental health and satisfaction with management scores between the two groups although our study showed that surgery or conservative treatment does not implement successful patient satisfaction.

Table 1

\begin{tabular}{|c|c|c|c|}
\hline Subgroups & Patients $(n=80)$ & Mean \pm SD & $P$ value \\
\hline \multirow[t]{2}{*}{ Pain } & Surgical treatment $(n=35)$ & $4.09 \pm 0.78$ & 0.376 \\
\hline & Conservative treatment $(n=45)$ & $3.92 \pm 0.61$ & \\
\hline \multirow[t]{2}{*}{ Function } & Surgical treatment $(n=35)$ & $3.83 \pm 0.87$ & 0.433 \\
\hline & Conservative treatment $(n=45)$ & $3.99 \pm 0.71$ & \\
\hline \multirow[t]{2}{*}{ Self-image } & Surgical treatment $(n=35)$ & $3.17 \pm 0.81$ & 0.558 \\
\hline & Conservative treatment $(n=45)$ & $3.31 \pm 0.01$ & \\
\hline \multirow[t]{2}{*}{ Mental health } & Surgical treatment $(n=35)$ & $3.08 \pm 0.98$ & 0.791 \\
\hline & Conservative treatment $(n=45)$ & $3.15 \pm 0.83$ & \\
\hline \multirow[t]{2}{*}{ Satisfaction with management } & Surgical treatment $(n=35)$ & $1.52 \pm 0.53$ & 0.612 \\
\hline & Conservative treatment $(n=45)$ & $1.45 \pm 0.55$ & \\
\hline
\end{tabular}

${ }^{1}$ Canakkale Onsekiz Mart University Physical Medicine and Rehabilitation Department, Canakkale, Turkey 


\section{Author details}

${ }^{1}$ Canakkale Onsekiz Mart University Physical Medicine and Rehabilitation

Department, Canakkale, Turkey. ${ }^{2}$ Physical Therapy and Rehabilitation School,

Istanbul, Turkey.

Published: 27 January 2012

doi:10.1186/1748-7161-7-S1-P15

Cite this article as: Yilmaz and Kuru: A comparison of results of SRS-30

questionnaire in scoliosis patients treated surgically or conservatively.

Scoliosis 2012 7(Suppl 1):P15.

Submit your next manuscript to BioMed Central and take full advantage of:

- Convenient online submission

- Thorough peer review

- No space constraints or color figure charges

- Immediate publication on acceptance

- Inclusion in PubMed, CAS, Scopus and Google Scholar

- Research which is freely available for redistribution

Submit your manuscript at 